\title{
Mutational Analysis in Gastrointestinal Stromal Tumors - A Series of Three Cases
}

\author{
Alexandra Roşulescu ${ }^{1}$, Adina Croitoru², Andreea lorgescu ${ }^{3}$, Gabriel Becheanu ${ }^{4}$, Alexandru Procop ${ }^{3}$, \\ Victor Tomulescu ${ }^{5}$, Vlad Croitoru ${ }^{6}$, Vlad Herlea ${ }^{7}$
}

Corresponding author: Vlad Herlea, MD, PhD Associate Professor of Pathology Department of Pathology Fundeni Clinical Institute Faculty of Medicine University "Titu Maiorescu" Sos. Fundeni 258 , sect.2 Bucharest, Romania E-mail: herlea2002@yahoo.com

\author{
'Department of Pathology, Fundeni Clinical Institute, Bucharest, Romania \\ Faculty of Medicine "Carol Davila", Bucharest, Romania \\ ${ }^{2}$ Department of Oncology, Fundeni Clinical Institute, Bucharest, Romania \\ Faculty of Medicine, University "Titu Maiorescu”, Bucharest, Romania \\ ${ }^{3}$ Department of Pathology, Fundeni Clinical Institute, Bucharest, Romania \\ ${ }^{4}$ Department of Pathology, Fundeni Clinical Institute, \\ University of Medicine "Carol Davila", Bucharest, Romania \\ ${ }^{5}$ Department of Surgery, Ponderas Hospital, Regina Maria, Bucharest Romania \\ ${ }^{6}$ Department of Internal medicine, Craiova County Hospital, Craiova, Romania \\ ${ }^{7}$ Department of Pathology, Fundeni Clinical Institute, \\ Faculty of Medicine, University "Titu Maiorescu", Bucharest, Romania
}

\section{ABSTRACT}

Background: Most of the Gastrointestinal Stromal Tumors (GISTs) are determined by mutations in C-KIT or PDGFRA genes. Mutation type influences the clinical evolution and treatment response.

Case reports: This study aims to present particular cases of GIST referred for molecular testing, diagnosed and treated in our institution.

Results: We present three cases in which genetic testing was performed. The first patient was diagnosed in a short-time interval with two GISTs, a gastric and a jejunal one, both with low risk of recurrence. To establish the relationship between the two, molecular analysis was performed and the conclusion was that we were facing synchronous, sporadic GISTs, a very rare instance. The second case was a patient with liver metastases, 3 years earlier having been diagnosed with a high risk duodenal GIST. The genetic test revealed a mutational status conferring imatinib sensitivity and therefore imatinib was continued. The third case was a patient with an early recurrence in within less than a year after surgery for gastric GIST. Molecular testing identified a mutation associated with a bad clinical outcome. The patient died 2 years later from diagnosis by disease progression.

Conclusion: Although rarely performed, genetic analysis provides useful information regarding the prognosis and the response to TKIs.

Key words: gastrointestinal stromal tumor, gene, mutation, exon, molecular analysis

\section{INTRODUCTION}

Gastrointestinal stromal tumors (GISTs) are the main subtype of mesenchymal tumors of the digestive tract, rare entities, originating in the interstitial cells of Cajal from the myenteric plexus. Frequently, the genetic event that triggers 
the tumorigenesis is represented by a mutation in c-KIT proto-oncogene. This discovery led to a better understanding of the pathologic process and to the development of targeted molecular therapy.

Up to $90 \%$ of adult GISTs harbour c-KIT or PDGFRA a homologous tyrosine kinase receptor -mutations. The remaining tumors are designated as Wild-type (WT) GISTs (1). Among these, other driving mutations were identified such as BRAF gene, NF1 gene, SDH complex. Approximately $5 \%$ of GISTs still don't have a recognised genetic alteration (2). KIT mutations present in GISTs determine ligand-independent activation of the receptor, promoting proliferation and growth. The most frequently involved regions are exons 9, 11, 13 and 17. The most common oncogenic mutations are in exon 11 and can take different forms, presenting themselves as deletions, substitutions, insertions or a combination of these (3). The majority of these mutations were identified in gastric GISTs and were associated with spindled morphology (4). Mutations in exon 9 represent, in almost all cases, duplications and were predominantly reported in tumors within the small and large bowel. Also they were associated with reduced sensitivity to imatinib and a higher mortality $(5,6)$. Mutations of exons 13 and 17 are very rare and their functional implications are yet to be determined (7).

Similarly to KIT, PDGFRA is a tyrosine kinase receptor whose mutation causes downstream activation of several signalling pathways involved in vital cellular functions. KIT and PDGFRA mutations are mutually exclusive in GISTs, a fact strengthened by their similar role. PDGFRA mutations were found in approximately $7 \%$ of GISTs, more than $80 \%$ being of missense type. They were more frequently identified as epithelioid type tumors located in the stomach, mesentery and omentum (8). Compared to KIT mutated GISTs, PDGFRA mutated ones have a lower risk of recurrence (9).

Although most of the KIT and PDGFRA mutations are somatic, in rare instances germ cells can become involved, resulting in an inherited predisposition to multiple GISTs development. The transmission is autosomal dominant and mutation types, as tumor morphology, are identical with sporadic cases. Cajal cell hyperplasia is an accompanying phenomenon and as a result tumors usually appear at younger ages (10).

$10-15 \%$ of GISTs are wild-type, and were recently described with the help of the molecular analysis as an heterogeneous group. Up to $40 \%$ of these cases are $\mathrm{SDH}$-deficient stromal tumors with loss-of-function mutations in any of the four SDH subunits: A, B, C or D and subsequent loss of subunit $B$ protein expression. The SDH-deficient GISTs have a similar pattern: are diagnosed at young ages, affect females more frequently, are localized in the stomach, have a mixed epithelioid and spindle cellular pattern, are KIT and DOG1 positive, with often lymph node metastases and an indolent course (11). This mutations appear in most pediatric GISTs, some are responsible for sporadic GISTs while other determine two syndromes: Carney triad (gastric GISTs, paragangliomas and pulmonary chondromas ) and Carney Stratakis syndrome (gastric GISTs and paragangliomas) (12). Neurofibromatosis type $I$ is an autosomal dominant genetic disorder in which among other tumors, multiple GISTs can arise. NF1 related GISTs are spindle cell type, develop skenoid fibers and affect the small bowell. KIT or PDGFRA mutations are absent (13). Up to $13 \%$ of WT GISTs harbour V600E BRAF mutations. These tumors arise most frequently in the small intestine, in middle-aged females, have a high mitotic rate, metastasize early in the course of the disease and respond to BRAF inhibitors (14).

Additionally, other somatic mutations have been discovered in GISTs, including in genes as PIK3CA, MAX, FGFR1, TP53, APC, MEN1 etc. Although found in a limited number of cases, these mutations demonstrate the great molecular heterogeneity of GISTs, fact also reflected by the large spectrum of clinical manifestations of these neoplasms (15).

A positive diagnosis of GIST is currently strengthened with the help of immunohistochemistry, c-KIT and DOG1 being highly sensitive and specific markers. The role of mutational analysis in this tumor type management is still a matter of debate and standardisation lacks. An algorithm of complete GIST diagnosis is ilustrated in fig 1 (16).

\section{CASE REPORTS}

In our practice a limited number of patients with GISTs were referred for genetic testing and hereby we report our experience regarding the use of this analysis in the following cases. These particular investigations could not be performed in our hospital and all the cases presented here were selected from the files of the patients who returned at the Pathology Department with the results from private laboratories, that were initially treated and diagnosed in our medical institution.

\section{Case 1}

A 61-year-old male patient without significant history, with occasional gastroesophageal reflux, was diagnosed with an extramucosal gastric mass on ultrasound, in the course of a routine exam. The 


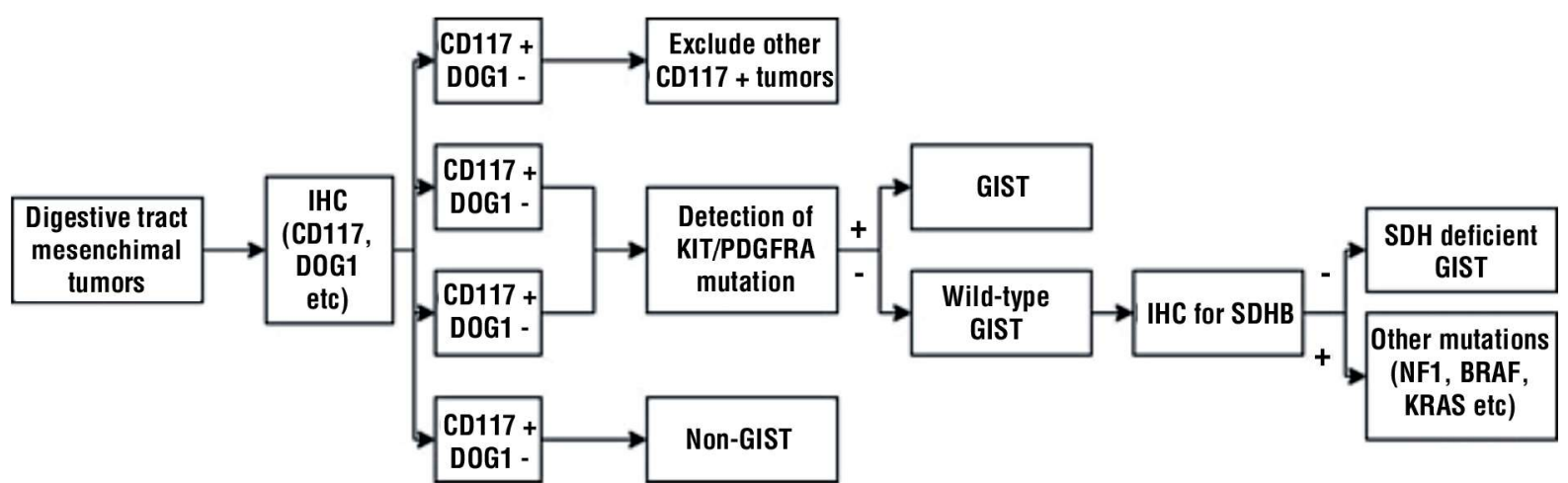

Figure 1 - Algorithm of GIST diagnosis

(simplified after Chinese consensus guidelines of diagnosis and management of gastrointestinal stromal tumor)

diagnosis was confirmed by endoscopy, computed tomography with intravenous contrast and echoendoscopy. The lesion was described as a circumscribed, nodular mass, located in the posterior wall of the antrum, with a maximum diameter of $2.6 \mathrm{~cm}$, hypoechoic on ultrasound and spontaneously hypodense, with intense, progressive iodophilia on CT. (fig. 2 a) Subsequently, a surgical intervention was performed for the removal of the tumor using robotic approach, with a favourable postoperative course. Pathological report described a nodular, elastic mass, measuring $2.3 \mathrm{~cm}$ post fixation, with a white-grey color. Microscopic exam revealed a mesenchymal proliferation of spindle cells, with a storiform pattern, little pleomorphism, perinuclear vacuolisations and rare mitotic figures (3/50 HPF), suggesting the diagnosis of GIST with low risk of disease progression, according to National Institutes of Health (NIH) criteria (fig. 2 b) Immunohistochemistry showed CD117, DOG1, CD34, SMA and Vimentin positivity, and the proliferation index Ki67 was as low as $5 \%$, confirming the histopathological diagnosis.

3 months after the surgery, in the course of a postoperative evaluation, a second tumor mass was discovered on computed tomography, at the level of the jejunal wall with both intra and extraluminal components, measuring $2.1 \mathrm{~cm}$ in maximum diameter, with solid features and described as nodular, circumscribed and homogeneous (fig. 2 c) No local recurrence was determined at the previous tumor site. Surgical removal of the tumor was performed by laparoscopy

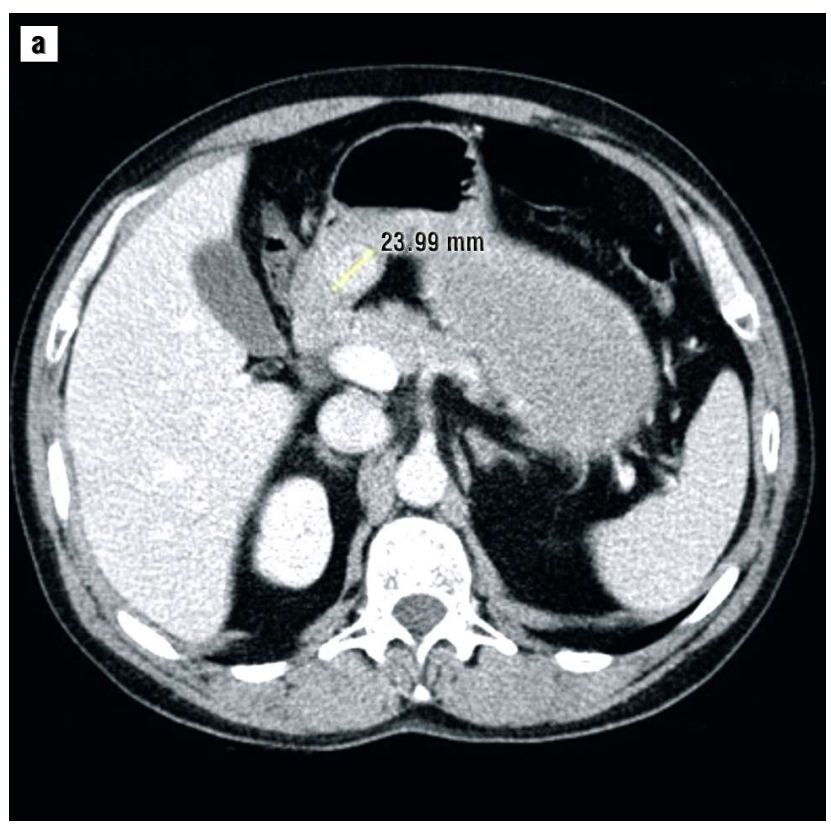

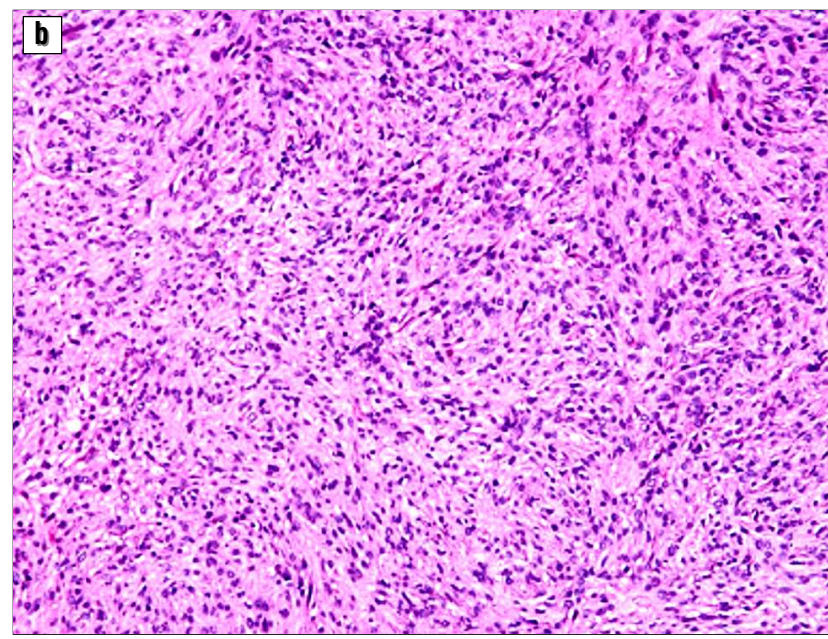

Figure 2 - Imagistic and histopathological aspects of case 1 tumors a) CT scan shows an intramural, round, solid tumor mass at the level of the gastric antrum.

b) Gastric GIST presenting as uniform, dense proliferation of spindle cells arranged in whorls with low mitotic rate (HE 20x) 

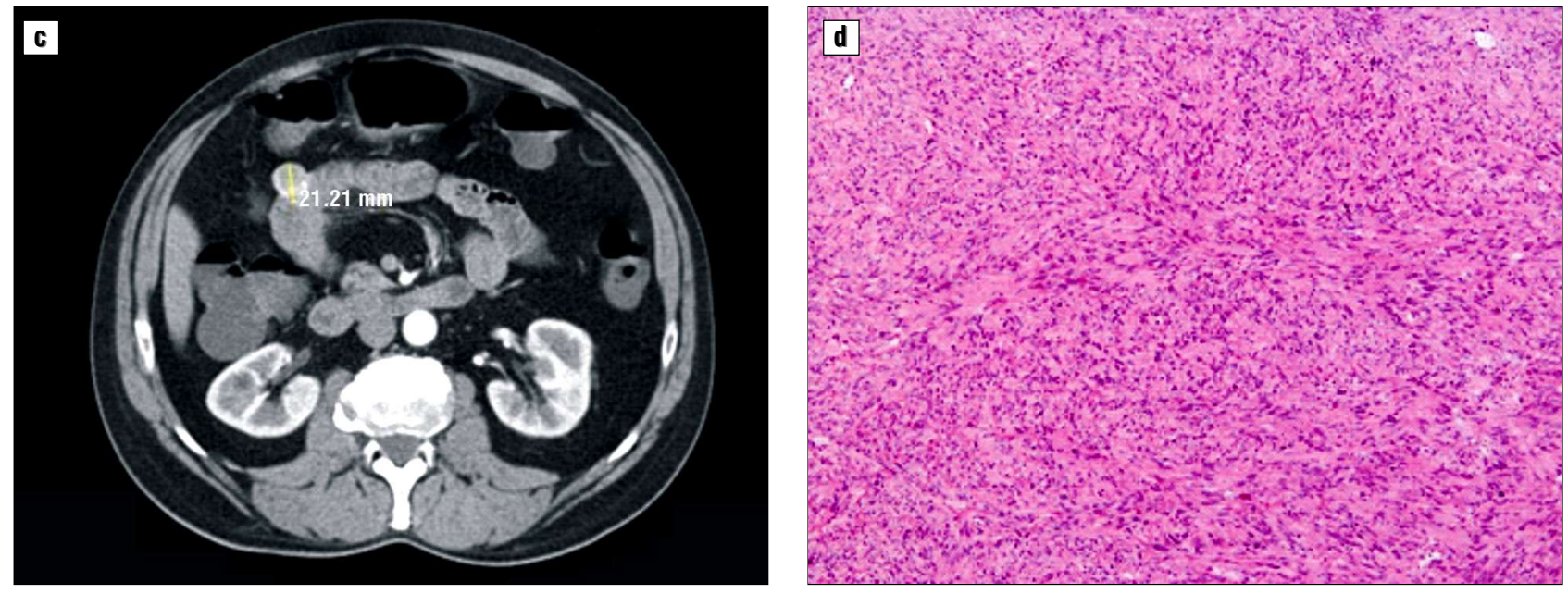

Figure 2 - Imagistic and histopathological aspects of case 1 tumors

C) CT scan reveals' a round, solid tumor mass attached to the jejunal wall with endo- and extraluminal expansion.

d) Jejunal GIST consisting of a monomorphic proliferation of spindle cells arranged in fascicles with a low mitotic rate (HE 10x).

with a favourable clinical outcome. Macroscopically, the tumor measured $2 \mathrm{~cm}$, had an elastic consistency and a white-yellowish colour. The histopathological aspect revealed a dense cellular proliferation of spindle type with a fascicular pattern, reduced pleomorfism and low mitotic rate (2 mitoses/50 HPFs), with frequent skenoid fibers (fig. $2 d$ ).

On immunohistochemistry, tumor cells were CD117, DOG1, S100, Vimentin positive, CD34 and Desmin negative, and Ki 67 proliferation index was less than $5 \%$. The criteria for the diagnosis of GIST with very low risk of disease progression were met under these circumstances.

Considering that the diagnosis of two similar GISTs was made in the same patient at a short time interval, a metastatic instance had to be excluded and molecular analysis was performed in search for mutations in C-KIT (exons 9,11,13,18 and 20) and PDGFRA (exons 12 and 18) genes in both tumors. In the gastric tumor a deletion in KIT exon 9 (c.1450_1461del12, p.K484_ G487del) was detected while in the jejunal tumor a single nucleotide polymorphism in PDGFRA exon 18 was identified. The discovery of different genetic profiles, supported by different histopathological and immunohistochemical aspects, led to the conclusion that the two GISTs were synchronous, sporadic tumors, a metastatic, familial or syndromic instance being excluded. Consequently, no adjuvant therapy was administered as surgery is the only treatment in such sporadic cases and the patient had a favourable clinical course.

The particularity of this case consists in the existence of two sporadic and also synchronous GISTs, with dissimilar locations, a gastric and an intestinal one and different genetic alterations, both having a low risk of disease progression, an instance rarely reported.

\section{Case 2}

A 61-year-old male patient with no significant family or personal history, was diagnosed with a tumor mass involving the duodenum and extending towards the jejunum and the uncinate process of the pancreas. An extensive surgical intervention was performed with tumor resection en-bloc with cephalic duodenum pancreatectomy, extensive dissection from the superior mesenteric vascular pedicle, cholecystectomy and multiple biliary-digestive anastomoses. At gross examination the tumor had solid and cystic areas and a diameter of $14 \mathrm{~cm}$. Microscopically, a mesenchymal proliferation was observed, being composed of mixed type cells, spindle and epithelioid with moderate pleomorphism, necrosis, haemorrhage and a low mitotic rate of less than 5/50 HPF (fig. 3 a). Superjacent mucosa was infiltrated and ulcerated and the pancreatic parenchyma was only compressed, with no sign of tumor invasion. Immunostaining for CD117, SMA and Vimentin was positive, for desmin was negative and Ki 67 index was about $10 \%$. The final diagnosis was GIST with high risk of disease progression according to NIH criteria. Under these circumstances, the patient had an indication for adjuvant treatment and imatinib therapy was initiated.

Periodical follow-up exams showed no signs of disease in the following years. An MRI scan performed after 3 years revealed three hepatic masses in segments II, IVA and VII, measuring between 0.6 and $1.5 \mathrm{~cm}$, consistent with metastatic disseminations. Shortly 

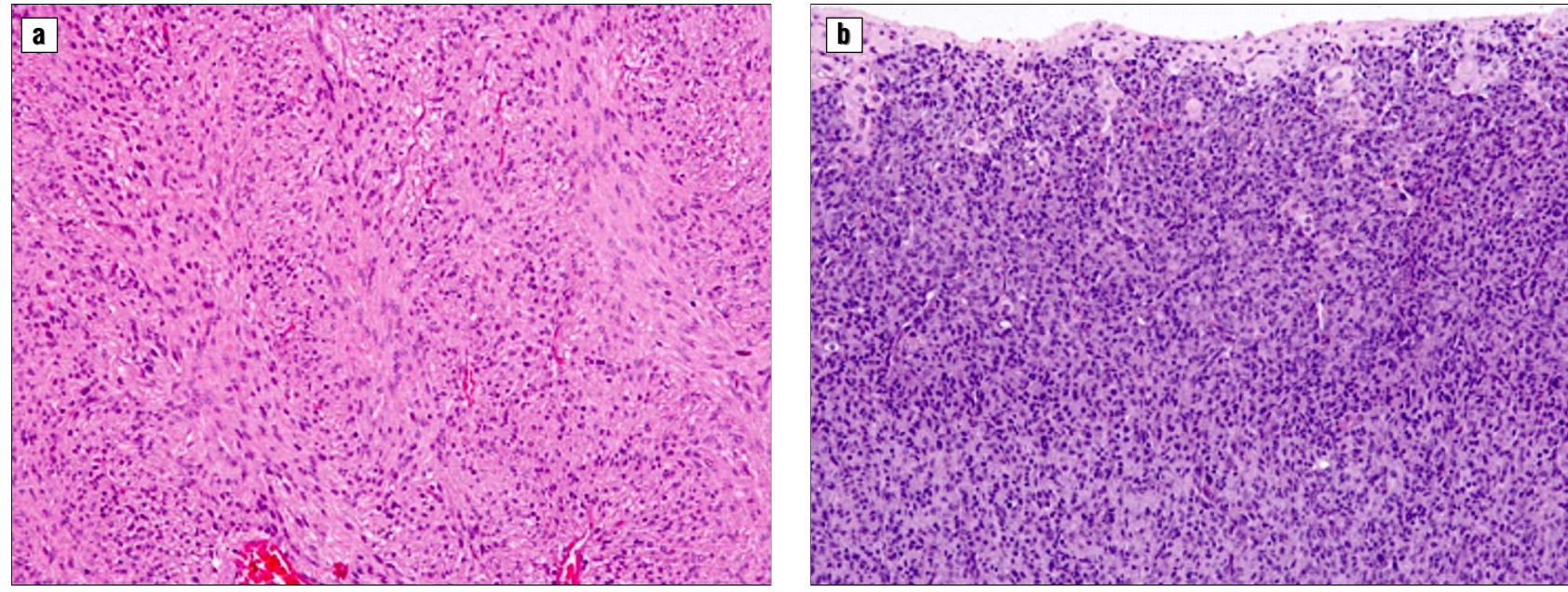

Figure 3 - Histopathological aspects of case 2 tumors. a) Duodenal GIST: spindle cells proliferation with fascicular arrangement and a low mitotic rate (HE 20x). b) Hepatic metastasis of GIST: dense, epithelioid cells proliferation with frequent mitotic figures (HE 20x)

thereafter, the tumors were surgically removed under echography control. The pathology report described nodular, whitish tumor masses composed predominantly of pleomorphic, epithelioid cells with areas of hyalinization and myxoid change, with a high mitotic rate (>5/50 HPF) (fig. 3 b). Immunohistochemical testing revealed CD117, DOG1 and CD34 positivity and a Ki67 index of $30 \%$, sustaining the diagnosis of hepatic metastases of GISTs. Also, mutational analysis was performed for the secondary tumors with the identification of an insertion (c.1673 1674insTCC) at the level of exon 11 of C-KIT gene. Considering the genetic test result, exon 11 mutations being known to have good responses to TKI therapy according to evidence, imatinib treatment continuity was indicated, with no signs of disease progression at follow-up, about 5 years after the initiation of the medication.

This case emphasises the role of mutation analysis in treatment guidance. Studies show that generally exon 11 mutated tumors are sensitive to Imatinib and although the patient developed metastases, he can still benefit from this therapy.

\section{Case 3}

A 40-year-old male patient presented with mesogastric area and referred back pain, nausea and weight loss. The CT scan revealed a tumor mass involving the stomach, the pancreas and the spleen, measuring $17.8 / 12.6 \mathrm{~cm}$, with an irregular contour composed of solid and cystic areas. Gastric endoscopy report described a submucosal mass extending from the cardia to the antrum, with reduced gastric cavity. The tumor was surgically removed by total gastrectomy en-bloc with corporeo-caudal spleno-pancreatectomy and omentectomy, followed by eso-jejunal anastomosis. On gross examination, the tumor was located in the thickness of gastric wall and infiltrated the spleen and the pancreas, had a grey-white colour and elastic consistency. Microscopically, the tumor cells were predominantly spindle shaped, rarely epithelioid, with fascicular arrangement, had hyperchromatic nuclei and areas of necrosis. The mitotic rate was high ( $>5$ mitoses (50 HPF), also with atypical figures ( $f$ ig. $4 \mathrm{a}$ ). Immunohistochemistry revealed CD117, DOG1, CD34 positivity, S100 and actin were negative and Ki67was positive in $50 \%$ of cells. In conclusion, the diagnosis was GIST with high risk of recurrence. Surgical margins and resected lymph nodes were tumor free. As European Society for Medical Oncology (ESMO) and European Sarcoma Network Group clinical practice guidelines indicate adjuvant imatinib for high risk tumors, imatinib treatment was initiated (17).

10 months after surgery, the patient complained of left upper quadrant pain and the CT scan revealed the recurrence of the tumor in the gastric compartment, measuring $11.7 / 8 \mathrm{~cm}$. A second surgery was performed with the tumor removal accompanied by segmental jejunal and colonic splenic flexure resection, structures infiltrated by the neoplasm. Also, tumor nodules of the mesentery, left adrenal gland and peritoneum were identified and excised. On gross inspection the tumor had a maximum diameter of $14 \mathrm{~cm}$, had a gray-white color with areas of necrosis and haemorrhage. Histopathologically, a diagnosis of GIST metastases was confirmed, with a pleomorphic, mixed: epithelioid and spindle cellularity and a very high mitotic rate $(>20$ 

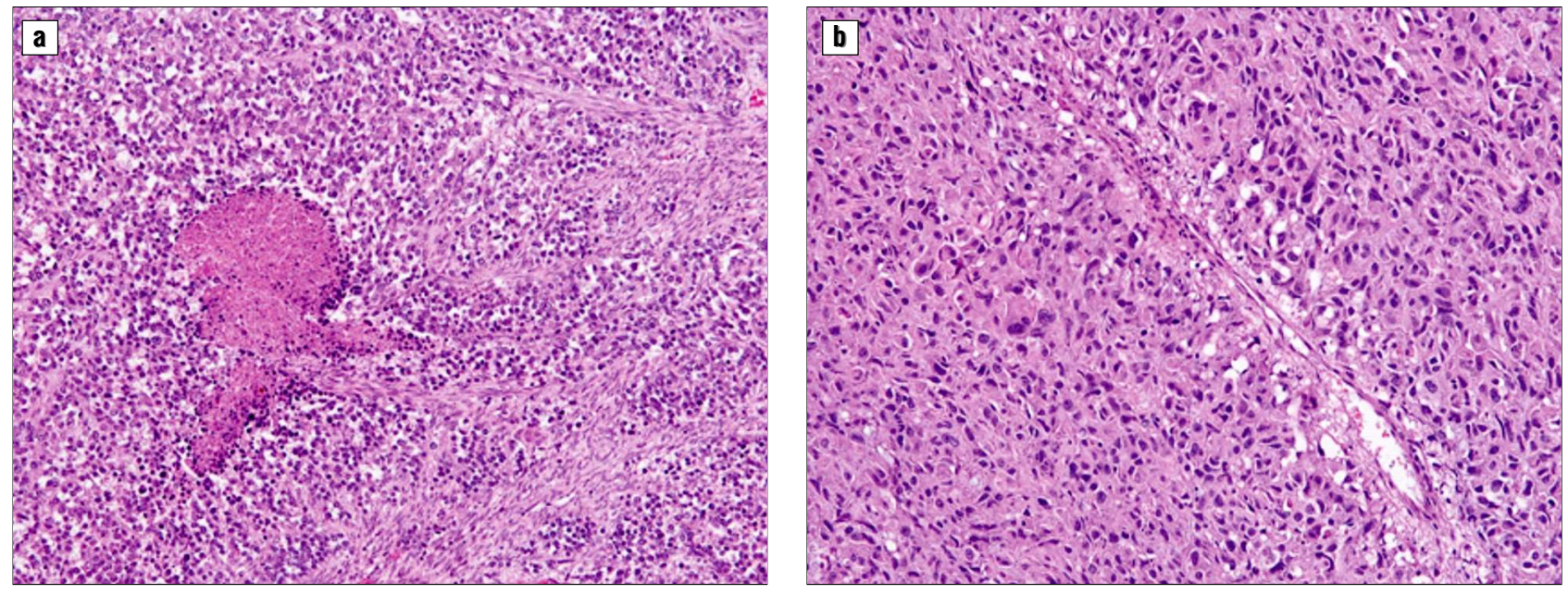

Figure 4 - Histopathological aspects of case 3 tumors. a) Gastric GIST: mixed cell morphology: spindle and epithelioid with an area of necrosis (HE 10x); b) GIST relapse: pleomorphic epithelioid cells proliferation (HE 20x)

mitoses/10 HPF) (fig. 4 b). Considering a lack of response to the Imatinib, mutational analysis was recommended and an in frame insertion was identified in exon 9 of C-KIT gene (c.1509_1510insGCCTAT), confirming that treatment resistance has a molecular basis. Afterwards, the patient chose to be evaluated and treated in another medical care unit abroad but, approximately 15 months after the second surgical intervention, he died. Details about the alternative therapeutic approach were not available. This case emphasises, once again the major role of the precise genetic alteration in the determinism of tumor prognosis. Exon 9 mutations are known to have a dismal evolution and our patient did not represent an exception, proof being his resistance to adjuvant treatment and tumor related death.

\section{DISCUSSION}

The discovery of kit mutations at the base of GISTs revolutionised the treatment for these tumors which were chemo and radiotherapy resistant. Tyrosine kinase inhibitor imatinib was developed as a treatment for chronic myelogenous leukaemia for its capacity of inhibiting the oncoprotein BCR-ABL (18). After structural similarities were observed between $A B L$ and $\mathrm{KIT}$, experiments proved imatinib to be also a potent inhibitor for KIT, disease control being achieved in a significant percentage of advanced GISTs immunohistochemically positive for c-kit, with a great increase of disease free and overall survival (19). A subset of the patients treated with imatinib develop resistance due to secondary mutations. In this situations, alternative TKI (tyrosine kinase inhibitors) proved to be effective such as sunitinib, sorafenib, regorafenib, nilotinib, dasatinib etc (20). A key role in the response to imatinib (or alternatives) treatment is played by the precise mutation determining the tumorigenesis. Patients with exon $11 \mathrm{KIT}$ mutations, as in case 2, proved to have a longer disease free and overall survival than those with exon 9 - as 3rd case described - or those of wild-type (21). Identifying these mutations can predict the response to treatment and makes possible a personalised treatment regarding the type of TKI and the dose (22).

A positive pathologic diagnosis of GIST is made on morphology, spindle cell type being predominant, in $70 \%$ of cases, followed by epithelioid cell type (20\%) and mixed cell type (10\%) completed by immunohistochemical testing for CD117 (c-KIT) that is positive in 95\% of cases and/or DOG1, with high sensibility and specificity; CD34 is also expressed in approximately $70 \%$ of cases and in a smaller percentage of tumors SMA, S100, desmin or h-caldesmon (23).

At the moment, molecular diagnosis in GISTs is recommended by National Comprehensive Cancer Network (NCCN) for high-risk surgical resected tumors, as defined by NIH criteria, that takes into account tumor size and mitotic rate (24). As for risk assessment, GISTs have a great variability when it comes to their clinical behaviour: they can be indolent, when incidentally diagnosed or they can be highly malignant tumors, resections being followed by local recurrences or distant metastases, with high morbidity and mortality. As small GISTs and low mitotic rate GISTs can rarely metastasize, a clear cut-off between benign and malignant tumors as such could not be established. A more feasible approach was to establish risk categories. $\mathrm{NIH}$ consensus defines high risk tumors those with a 
diameter greater than $5 \mathrm{~cm}$ and a mitotic rate above $5 / 50 \mathrm{HPF}$ or a diameter greater than $10 \mathrm{~cm}$ and any mitotic rate or any dimension and a mitotic rate greater than 10/50 HPF (25).

The anatomic location of the tumor is another important variable, being associated with a more frequent type of mutation and, as a result, with a different response to treatment and a different prognosis. Although gastric and oesophageal GISTs have smaller size and lower mitotic rate at diagnosis, associated with a better overall prognosis, the response to imatinib seems to be better in patients with small intestinal GISTs (26).

Imatinib is the first-line treatment for inoperable, metastatic, or recurrent GISTs. The standard dosage is $400 \mathrm{mg} /$ day but guidelines recommend a higher dosage for KIT exon 9 mutated GISTs because the higher dosage $(800 \mathrm{mg} /$ day) showed longer survival (27). Patients classified by AFIP (Armed Forces Institute of Pathology) as intermediate or high risk (above group $3 a)$ - with a 5-year recurrence rate of $30 \%$ - have a higher risk of recurrence and therefore should be taken into consideration for adjuvant therapy (28).

Eventually, the benefit of imatinib depends on one side of the mutation that drives the tumorigenesis and determines the sensitivity to the drug, and on the other side by the comorbidities that influence clinical outcome.

Apart from predicting the prognosis and response to treatment, the mutation analysis, as we showed in case 1 , can differentiate multiple sporadic tumors from metastatic instances by emphasising the distinct molecular events that triggered the neoplastic process. This has a great clinical significance, as stage and therapeutic approach are fundamentally different in the two different situations.

Undoubtedly, molecular testing can come with significant improvement in GISTs management but, unfortunately, despite proven benefits, it's still rarely done in current practice.

\section{CONCLUSION}

In conclusion, determining mutation type in GISTs can predict the response to treatment and clinical outcome, providing a useful tool in their management. Molecular characterisation is very important in identifying the patients with a high risk of disease relapse and should be mandatory for a personalised treatment.

Although a useful instrument, genetic testing in GISTs is far from becoming a routinely used investigation. A very limited number of cases, in our experience benefit from it. That is in part because of a lack of standardisation but mostly because of material issues and ineffective healthcare politics. Hopefully, further benefits evidence will contribute to an increase in exon mutation analysis in the case of GISTs.

\section{Ethics committee approval}

Ethics committee approval was received for this study from the Ethics Committee of Fundeni Clinical Institute (20636/07.04.2020).

\section{Informed consent}

Informed writen consent was obtained from the involved patients, according to internal local procedures of Fundeni Clinical Institute.

Conflict of interest: none to declare.

\section{Financial disclosure}

This study has received no financial support.

\section{Author's contributions}

Alexandra Rosulescu and Adina Croitoru = equal contribution.

\section{REFERENCES}

1. Corless CL, Fletcher JA, Heinrich MC. Biology of gastrointestinal stromal tumors. J Clin Oncol. 2004; 22:3813-25.

2. Patil DT, Rubin BP. Genetics of Gastrointestinal Stromal Tumors A Heterogeneous Family of Tumors? Surgical Pathology. 2015; 8: 515-524.

3. Mol CD, Dougan DR, Schneider TR, Skene RJ, Kraus ML, Scheibe DN et al. Structural basis for the autoinhibition and STI-571 inhibition of c-Kit tyrosine kinase. J Biol Chem. 2004; 279:31655-31663.

4. Wozniak A, Rutkowski P, Schoffski P, Ray-Coquard I, Hostein I, Schildhaus $\mathrm{HU}$ et al. Tumor genotype is an independent prognostic factor in primary gastrointestinal stromal tumors of gastric origin: a European multicentre analysis based on ConticaGIST. Clin Cancer Res. 2014; 20:6105-6116.

5. Yuzawa S, Opatowsky Y, Zhang Z, Mandiyan V, Lax I, Schlessinger J. Structural basis for activation of the receptor tyrosine kinase KIT by stem cell factor. Cell. 2007; 130:323-334.

6. Heinrich MC, Owzar K, Corless CL, Hollis D, Borden EC, Fletcher CD et al. Correlation of kinase genotype and clinical outcome in the North American Intergroup Phase III Trial of imatinib mesylate for treatment of advanced gastrointestinal stromal tumor: CALGB 105105.Study by Cancer and Leukemia Group B and Southwest Oncology Group. J Clin Oncol. 2008; 26: 5360-5367.

7. Lasota J, Corless CL, Heinrich MC, Debiec-Rychter M, Sciot R, Wardelmann $\mathrm{E}$ et al. Clinicopathologic profile of gastrointestinal stromal tumors (GISTs) with primary KIT exon 13 or exon 17 mutations: a multicentre study on 54 cases. Mod Pathol. 2008; 21:476-484.

8. Heinrich MC, Corless CL, Duensing A, McGreevey L, Chen CJ, Joseph $\mathrm{N}$, et al. PDGFRA activating mutations in gastrointestinal stromal tumors. Science. 2003; 299:708-10.

9. Barnett $\mathrm{CM}$, Corless $\mathrm{CL}$, Heinrich MC. Gastrointestinal stromal 
tumors: molecular markers and genetic subtypes. Hematol Oncol Clin North Am. 2013; 27:871:888.

10. Kleinbaum EP, Lazar AJ, Tamborini E, Mcauliffe JC, Sylvestre PB, Sunnenberg TD, et al. Clinical, histopathologic, molecular and therapeutic findings in a large kindred with gastrointestinal stromal tumor. Int J Cancer 2008; 122:711-8.

11. Nannini M, Urbini M, Astolf A, Biasco G, Pantaleo MA. The progressive fragmentation of the KIT/PDGFRA wild-type (WT) gastrointestinal stromal tumors (GIST). J TransI Med. 2017; 15:113.

12. Gill AJ, Benn DE, Chou A, Clarkson A, Muljono A, Meyer-Rochow GY et al. Immunohistochemistry for SDHB triages genetic testing of SDHB, SDHC and SDHD in paraganglioma-pheochromocytoma syndromes. Hum Pathol. 2010; 41:805-814.

13. Takazawa Y, Dakurai S, Sakuma Y, Ikeda T, Yamaguchi J, Hashizume $Y$ et al. Gastrointestinal stromal tumors of neurofibromatosis type I (von Recklinghausen's disease).Am J Surg Pathol. 2005; 29:755-63.

14. Agaimy A, Terracciano LM, Dirnhofer S, Tornillo L, Foerster A Hartmann $A$ et al. V600EBRAF mutations are alternative early molecular events in a subset of KIT/PDGFRA wild-type gastro-intestinal stromal tumors. J Clin Pathol. 2009; 62:613-616.

15. Mei L, Smith SC, Faber AC, Trent J, Grossman SR, Stratakis CA et al. Gastrointestinal Stromal Tumors: The GIST of Precision Medicine. Trends in Cancer. 2018; 4: 74-91.

16. Li J, Ye Y, Wang J, Zhang B, Qin S, Shi Y et al. Chinese consensus guidelines for diagnosis and management of gastrointestinal stromal tumor. Chi J Cancer Res. 2017; 29(4):281-293.

17. Casali PG, Abecassis N, Bauer S, Biagini R, Bielack S, Bonvalot $S$ et al. Gastrointestinal stromal tumors: ESMO-EURACAN Clinical Practice Guidelines for diagnosis, treatment and follow-up. Annals of Oncology. 2018; 29(Supplement 4): 68-78.

18. Corless CL. Gastrointestinal stromal tumors: what do we know? Modern Pathology. 2014; 27: S1-S16.
19. Demetri GD, von Mehren $M$, Blake $C D$, Van den Abbeele $A D$, Eisenberg B, Roberts PJ et al. Efficacy and safety of imatinib mesylate in advanced gastrointestinal stromal tumos. N Engl J Med 2002; 347: 472-480.

20. Demetri GD, Differential properties of current tyrosine kinase inhibitors in gastrointestinal stromal tumors. Semin Oncol. 2011; 38:S10-S19.

21. Heinrich MC, Corless CL, Demetri GD, Blanke CD, von Mehren M, Joensuu $\mathrm{H}$ et al. Kinase mutations and imatinib response in patients with metastaticgastrointestinal stromal tumor. J Clin Oncol. 2003; 21:4342-4349.

22. Debiec-Rychter M, Sciot R, Le Cesne A, Schlemmer M, Hohenberger $P$, van Oosterom AT et al. KIT mutations and dose selection for imatinib in patients with advanced gastrointestinal stromal tumours. Eur J Cancer.2006; 42:1093-1103.

23. Nishida T, Blay JY, Hirota S, Kitagawa Y, Kang YK. The standard diagnosis, treatment, and follow-up of gastrointestinal stromal tumors based on guidelines. Gastric Cancer.2016; 19:3-14.

24. Von Mehren M, Randall L, Benjamin R, Boles S, Bui M, Ganjoo K et al. Soft tissue sarcoma, version 2.2018. Clinical practice guidelines in oncology. J Natl Compr Canc Netw. 2018; 16(5):536-563.

25. Joensuu $H$. Risk stratification of patients diagnosed with gastrointestinal stromal tumor. Hum Pathol. 2008; 39:1411-1419.

26. Valsangkar N, Sehdev a, Misra S, Zimmers TA, O'Neil BH, Koniaris LG. Current management of gastrointestinal stromal tumors: surgery, current biomarkers, mutations and therapy. Surgery. 2015; 158 (5): 1149-1163.

27. Gastrointestinal Stromal Tumor Meta-Analysis Group. Comparison of two doses of imatinib for the treatment of unresectable or metastatic gastrointestinal stromal tumors: a meta-analysis of 1640 patients. J Clin Oncol. 2010; 28:1247-53.

28. Balachandran VP, DeMatteo RP. GIST tumors: Who should get imatinib and for how long? Adv Surg. 2015; 48(1): 165-183. 